\title{
Building Multi-Material components by Direct Laser Deposition
}

\author{
Matteo Perini ${ }^{1,2,}$, Sasan Amirabdollahian ${ }^{1,2}$, and Paolo Bosetti ${ }^{1,2}$ \\ ${ }^{1}$ University of Trento, Department of Industrial Engineering, via Sommarive, 9 - 38123 Trento, Italy \\ ${ }^{2}$ ProM Facility, Via Zeni, 8 - 38068 Rovereto, Italy
}

\begin{abstract}
Most mechanical components are usually made of a single material, as a compromise between chemical, physical and functional properties. When designing an object, the choice of one material over another is driven by many reasons. Sometimes these reasons are not strictly technical, including for instance: biocompatibility, density, weldability, corrosion resistance, price, and appearance. Direct Laser Deposition (DLD) technology is an additive manufacturing process that allows the construction of objects by depositing material layer by layer. With DLD, the metal powder comes out of a nozzle conveyed by a flow of inert gas and is melted by a laser beam. One of the advantages of this technology over other AM technologies (such as SLM) is that it is possible to vary the composition of the deposited material by simply changing the metal powder. It is clear that with DLD technology, the properties of different metallic materials deposited together in a single object can be exploited. With this work the authors present two real-life case studies, in which the DLD technology is used to build multi-material components. The first case concerns a gearbox component made of $\mathrm{C} 40$ steel that must work in a food environment and has therefore been completely covered with a layer of stainless steel. The second case is a flange that must have a weldable base, while the internal hole must be made of a high-performance tool-steel.
\end{abstract}

\section{Introduction}

Each material has specific characteristics that make it more or less suitable for certain purposes. During the design of mechanical components, it often happens that the required specifications can only be partially met by a single material. To overcome this problem it often happens that different materials are combined together through traditional operations - e.g. welding, interference, riveting, screwing, adhesives, etc.

With the advent of the Direct Laser Deposition (DLD) technique, a new way of integrating different materials into a single component is opened up [1-3]. In recent years this technology has been integrated into 5-axis CNC machines - e.g. DMG MORI Lasertec $653 \mathrm{D}$ [4] - creating hybrid machines that allow both the addition and removal of material. Recently, the possibility of having multiple powder containers on board of the same machine has been implemented. This has made it possible to join one material to

\footnotetext{
*Corresponding author: matteo.perini@unitn.it
} 
another in a very simple way. It is also possible through the simultaneous use of the two powder containers to mix different materials. This way it is possible to change continuously the composition of the added material while working. In the two case studies presented in this work, only one material was deposited at a time.

With the DLD technique applied to CNC machines, the production process is much simpler. The main steps of the production process are:

1. modelling with a CAD software the geometry to be made

2. calculation of toolpaths using CAM software

3. addition of material through the hybrid machine

4. removal of surplus material by milling (optional)

One of the most interesting advantages of this technology is that the construction of the component happens using a single machine (hybrid) [4]. In addition, thanks to the use of lasers, it is possible to use materials that are usually difficult to weld or machine e.g. Tungsten Carbide (WC), Stellite etc. This technology also has its drawbacks, such as the heat input from the laser that could generate thermal stresses or distortions. Another shortcoming in the current hybrid machines is the lack of controlled atmosphere in their build space. Therefore, the deposition of materials that are prone to oxidation - e.g. Titanium and Aluminium alloys, is not possible in the hybrid machines available on the market. Many tests have been conducted to find the correct processing parameters for the deposition operations. The main physical parameters that can be manipulated on the machine are: the powder flow rate, the nozzle speed and the laser power. Other parameters such as the stepover, path-overhang and deposition-width can be set using CAM. Some of these parameters are closely interlinked and finding the best combination is a complex task $[5]$.

The paper explains the procedure applied to build a multi-materials component then, in Section 3 and 4, two real cases are described. Finally, in Section 5 and 6 results are analysed and discussed.

\section{The procedure}

The operations required to build a component [6] can be subdivided into two steps: the first one is composed by CAD/CAM operations while the second one is the actual manufacturing of the component through $\mathrm{CNC}$ machines.

The CAD design for additive operations presents some peculiarities because of the strategy adopted to depose different materials. The multi-material component must be created as a union of several sub-volumes i.e. one or more for each material. The contact areas between these volumes represent the interface surfaces between different materials. Between one AM operation and another, the interface areas will be reworked to provide a precise substrate for the subsequent additive operation. Moreover, solid models of the AM volumes must be offset so that sufficient material is deposed for the subsequent finishing of surfaces. To plan both AM and milling operations, it is therefore necessary to have solid CAD models of both the volumes.

When all solid models have been prepared, toolpaths can be calculated using a CAM software. Any CAM can be used for milling operations, while for the additive tasks the only software capable of managing DLD technology on the target machine is Siemens NX. Therefore, for convenience, Siemens NX was used for all operations.

Once the part-program has been created, the component can be built using the hybrid machine. The first step is to fix the starting component and reference it with respect to a coordinate system. In the eventuality that the whole component has to be realized through 
DLD technique, it is necessary to use a sacrificial layer on which the first layers of the component can be deposited. It is possible to add and subtract material alternately until the desired geometry is obtained.

\section{First test case}

The first object presented in this article - visible in Figure 1 - is a gearbox made of C40. This component must be covered with $316 \mathrm{~L}$ stainless steel because it must be used in a food environment.

The stainless steel ensures that, even in case of accidental contact with food, there is no contamination between them.
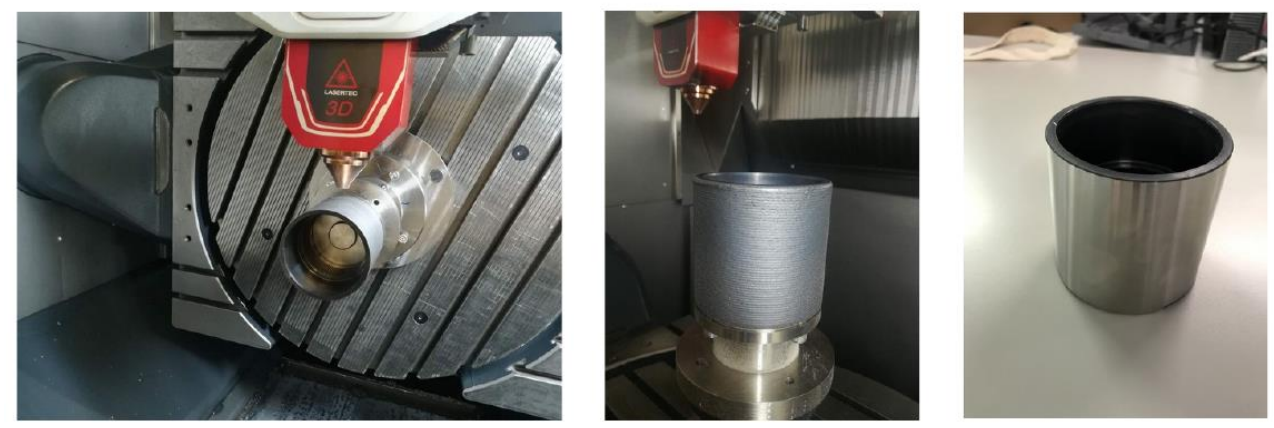

Fig. 1. From left to right: the additive operation, the cladded gearbox and the component after the finishing task

For the company that produces the gearbox, changing the material type of this component means stopping the production line - which normally works with the C40 - and adapting the machines and tools to the new material with a considerable waste of time and resources.

Moreover, the components made of stainless steel are only a fraction of the whole production.

The cladding of the external surface in this case appears to be a good solution to the problem of the company since it is convenient for small productions. A thin layer of stainless steel $316 \mathrm{~L}$ is sufficient to isolate the gearbox from food contacts and to prevent potential oxidation. Usually the component is painted to prevent surface corrosion. The paint should be removed completely to allow the stainless steel to adhere to the component. Following the workflow described in the previous section, it is possible to achieve the coverage of the external surface. The solid model of the component is expanded to represent the volume of the material to be added, then the toolpaths are created using the CAM software. Finally, the component is placed on the $\mathrm{CNC}$ machine and the new material is added. The process ends by turning or milling the component in order to restore its aesthetic appearance. To test the quality of the adhesion, the section of the worked area was analysed with metallography. Morover, to check the applicability of this method in the actual production of components, a complete dimensional inspection of the object was carried out. Three tests are performed: before the addition of material, after the additive operation (to check thermal distortions) and after the machining operation. To measure the component a coordinate measuring machine (CMM) was used.

\section{SECOND TEST CASE}

The second case study - visible in Figure 2 - is a flange for the automotive sector. 
The peculiarity of this object is that the central threaded bushing, visible in Figure X, must withstand heavy loads and is therefore made of a high-performance steel while the base of the flange must be tenacious and weldable so that it can be easily bonded to the chassis of the car. The union of these two components is difficult because of the bad weldability of the bushing. The interface between the two materials also creates a failure trigger that is not tolerable during normal use.

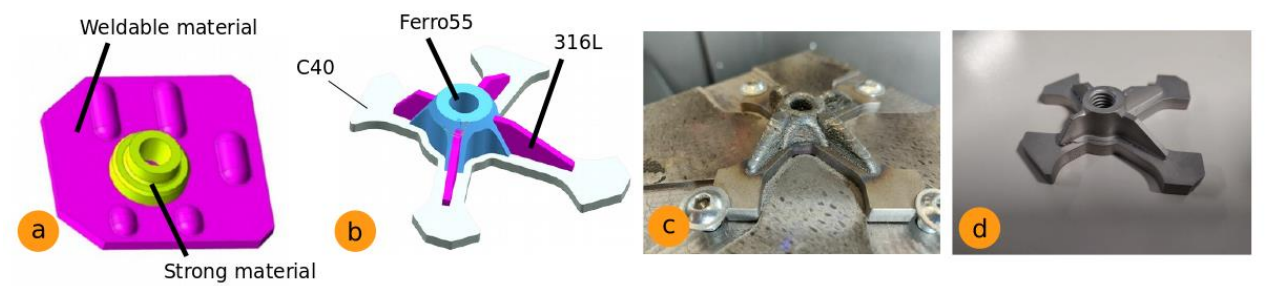

Fig 2. a) the original model composed of 2 parts, b) the new design for hybrid production, c) the final result after deposition, and d) the final part after milling operation.

The plate is also equipped with embossing in order to increase its stiffness. During a complete redesign of the component, the embossings have been replaced by gussets, which in addition to increasing the stiffness of the plate improve the bond between the bushing and the plate as well. Authors have designed the component starting from a laser-cut C40 steel base used as a substrate. On this base is deposited the bushing made of Ferro55, which is an equivalent to ASTM H13 tool steel and finally the gussets made in 316L stainless steel.

\section{Results and discussion}

The most important result is that, thanks to DLD technology, it was possible to solve the problems presented from the two case studies.
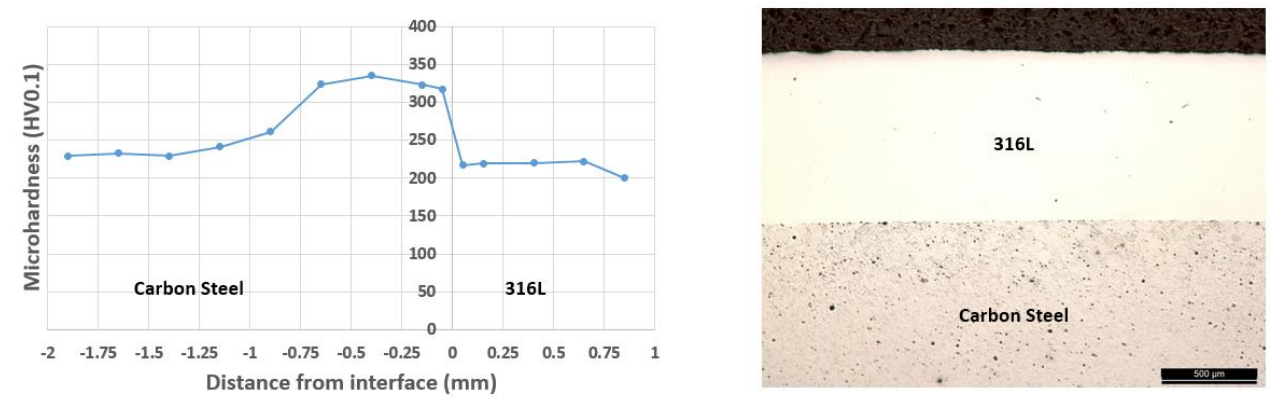

Fig. 3. Hardness profile on the left, cladding interface on the right

In the first case, the metallographic analyses carried out showed excellent continuity between the substrate and the added material. Figure 3 shows an image of the cladding interface and the relative hardness profile. The deformations due to thermal stress are contained and do not bring the component out of tolerance. In fact, the circularity goes from $0.030 \mathrm{~mm}$ to $0.045 \mathrm{~mm}$ after the cladding process, remaining inside the maximum tolerance limit of $0.05 \mathrm{~mm}$.

Gearbox manufacturing takes about 10 minutes for material addition and about 2 minutes for the finishing operation. This makes the cladding procedure convenient compared to the complete stainless steel fabrication. 
The second case study is more complex because it involves more materials and the geometries after the redesign are more complex than the previous case. In order to obtain a functional prototype, many tests have been carried out both with regard to the deposition parameters and to the toolpaths used. The authors looked for the DLD parameters that allow the material to be applied to the base without creating excessive deformations but ensuring, at the same time, the adhesion between the materials.

In this case study, the production of the component involves five additive operations: one for the central body and four for the gussets. The design of the central body has been adapted for the subsequent $\mathrm{CNC}$ machining operations. In particular, the central hole has been filled to facilitate drilling and the lateral surface has been made conical to facilitate the construction of the gussets.
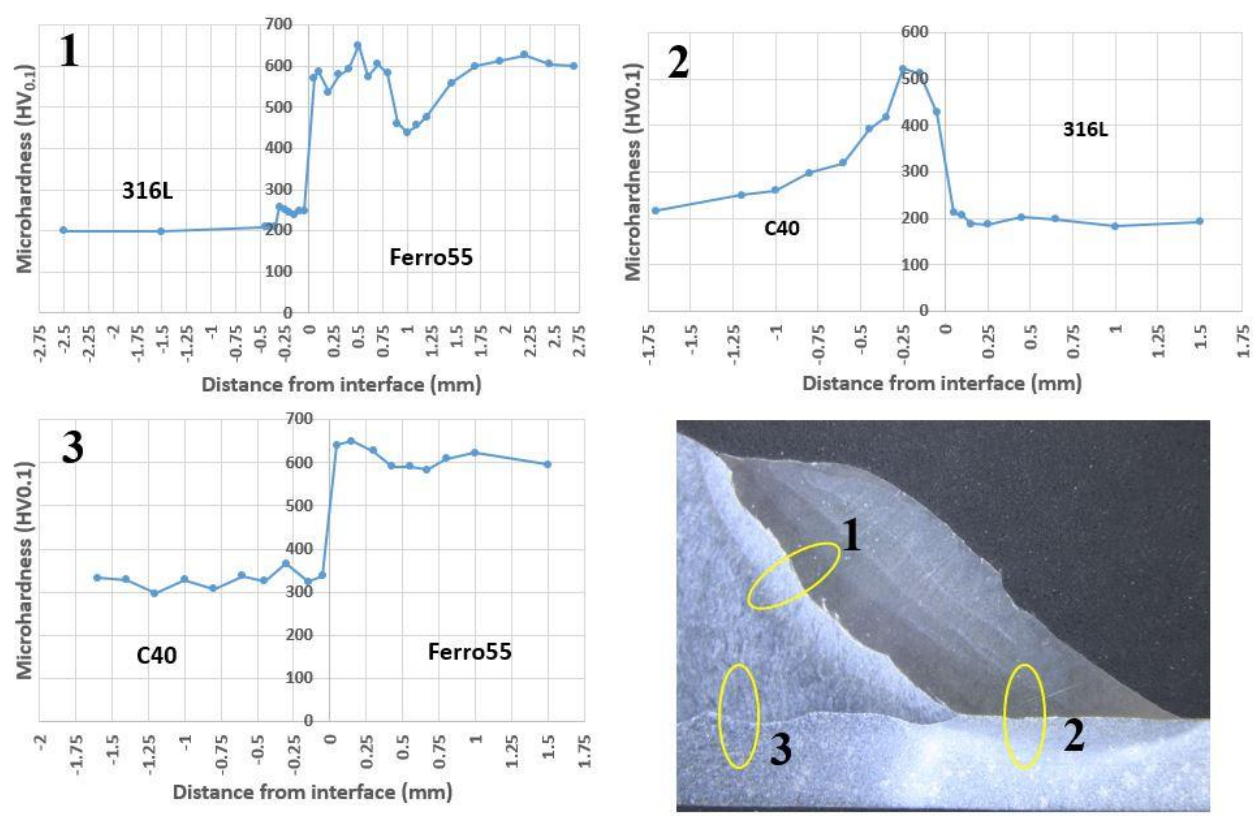

Fig. 4. Micrograph of a cross-section of the part and the relative hardness profiles for the three interfaces between different materials.

Figure 4 shows the hardness profiles for the interfaces among the three materials. It can be observed that for all of the three materials the microhardness in vicinity of the interface is higher with respect to the original material.

\section{Conclusions}

This work highlighted the advantages and critical points of the use of the DLD technique for the construction of multi-material components. This additive manufacturing method is very flexible and gives the real possibility to combine different materials while maintaining good performances. However, the use of the DLD, to be effective, should be motivated by both technical and economic reasons.

Moreover, using Direct Laser Deposition introduces heat into the component and, consequently, creates thermal stress and distortions that should be considered carefully. In the construction of complex shapes, the problem of accessibility of the areas to be worked 
must be considered, in fact, the DLD nozzle has large overall dimensions and a stand-off distance that limit the movement possibilities.

In both case studies, sound interfaces were obtained without observing any cracks or delamination. Moreover, by moving away from the interfaces the deposited materials showed similar properties to that of the bulk material, which means the material can satisfy the designed requirements.

However, alterations of the toolpaths and deposition parameters might lead to differences in microstructure, mechanical properties and adhesion of the materials, which will be investigated in future works in detail.

\section{References}

1. Thivillon, L., Bertrand, P., Laget, B., \& Smurov, I. Potential of direct metal deposition echnology for manufacturing thick functionally graded coatings and parts for reactors components. Journal of Nuclear Materials, 385(2), 236-241. (2009)

2. Mazumder, J., Schifferer, A., \& Choi, J. Direct materials deposition: designed macro and microstructure. Material Research Innovations, 3(3), 118-131. (1999)

3. Muller, P., Mognol, P., \& Hascoet, J. Y. Modelling and control of a direct laser powder deposition process for Functionally Graded Materials (FGM) parts manufacturing. journal of materials processing technology, 213(5), 685-692. (2013)

4. Ren, L., Sparks, T., Ruan, J., \& Liou, F. Integrated process planning for a multiaxis hybrid manufacturing system. Journal of Manufacturing Science and Engineering, 132(2), 021006. (2010)

5. Labudovic, M., Hu, D., \& Kovacevic, R. A three dimensional model for direct laser metal powder deposition and rapid prototyping. Journal of materials science, 38(1), 35-49. (2003)

6. Mazumder, J., Choi, J., Nagarathnam, K., Koch, J., \& Hetzner, D. The direct metal deposition of H13 tool steel for 3-D components. Jom, 49(5), 55-60. (1997) 